\title{
NON-INFECTIOUS GRANULOMATOUS ANGIITIS OF THE NERVOUS SYSTEM ASSOCIATED WITH HODGKIN'S DISEASE ${ }^{1}$
}

\author{
NEILL B. REWCASTLE AND MARY I. TOM \\ From the Division of Neuropathology, Department of Pathology, \\ University of Toronto, Canada
}

Neurological signs and symptoms associated with the granulomatous form of Hodgkin's disease are a well recognized entity, though infrequent. Invariably these are due to one of two pathological processes, by far the commonest being compression of the central nervous system from without, most frequently by an epidural mass within the spinal canal. An extremely rare occurrence is direct invasion of some part of the nervous system by Hodgkin's tissue. There have been many reviews of this subject (Ginsburg, 1927; Sparling, Adams, and Parker, 1947; Hutchinson, Leonard, Maudsley, and Yates, 1958). Winkelman and Moore (1941) emphasize the fact that the involvement of the central or peripheral nervous systems may occur as an initial manifestation of Hodgkin's disease.

In the following case, the clinical picture from onset to death was that of a progressive neurological disorder, late in the course of which the diagnosis of Hodgkin's disease was made by lymph node biopsy. At necropsy no invasion of the nervous system by Hodgkin's granuloma was found.

\section{CLINICAL HISTORY}

The patient, a 31-year-old white woman, was first admitted to hospital, 25 months before her death, as a result of two generalized convulsions. Each was followed by a short period of unconsciousness. The only positive findings were minimal hyper-reflexia of the left arm and a slight pyrexia. The cerebrospinal fluid contained 30 white blood cells per c. mm., chiefly lymphocytes, and $41.5 \mathrm{mg}$. \% of protein. No further convulsions occurred and she was discharged from hospital with the provisional diagnosis of lymphocytic meningo-encephalitis or suspected brain tumour.

During the ensuing four months she had several similar convulsions in spite of anticonvulsant therapy. Progressive numbness and weakness of the left arm were noted, which spread to involve the left leg. On her second admission to hospital, 21 months before death, there were, in addition, a dull constant ache over the

${ }^{1}$ Presented to the combined meeting of the sections of pathology and neurology and psychiatry, Academy of Medicine, Toronto, Canada, March 1960.

6 right frontotemporal region, and marked difficulty in walking. Examination revealed weakness and spasticity of the left side, most marked in the left arm, a questionable left Babinski response, and papilloedema of the right optic disc. The temperature was normal. A carotid arteriogram showed slight shift of the right anterior cerebral artery to the left, suggesting a space-occupying lesion in the right hemisphere, and air ventriculography showed a bulging downwards of the mid-portion of the roof of the right lateral ventricle. On this occasion the cerebrospinal fluid contained over 100 white blood cells per c. mm., again predominantly lymphocytes, and $\mathbf{1 8 0}$ mg. $\%$ of protein.

A needle biopsy from the lateral surface of the right frontal lobe produced small pieces of grey gelatinous tissue. Microscopic examination of this material revealed both cortex and white matter. Many small vessels showed all stages of obliteration by a proliferation of histiocyte-like cells with indistinct cytoplasmic boundaries and slightly irregular, elongated vesicular nuclei. In some instances, coalescence of these cells resulted in the formation of multinucleated giant cells. In the cortex, these granulomas were well circumscribed, and were producing secondary compression of adjacent parenchyma, the latter showing occasional degenerating nerve cells and hypertrophied astrocytes. In the white matter the granulomas were less well defined and showed a spilling over into the adjacent parenchyma of small round cells. The latter were considered to be lymphocytes, but the occasional plasma cell was also present. The adjacent parenchyma showed extensive oedema, numerous microglial cells, swelling and irregularity of myelin sheaths, and hypertrophied astrocytes. These changes are seen in Figs. 1 and 2, and were interpreted as evidence of a granulomatous encephalitis. Special stains failed to elicit any aetiological cause for this reaction.

Two weeks post-operatively there was a marked improvement in her condition without any specific therapy, and she was discharged to a rehabilitation and physiotherapy centre with slight left foot drop and some loss of position sense on the left side. On the above and subsequent admissions, intensive investigation was instigated as a result of the diagnosis of granulomatous encephalitis. All the following investigations were found to be negative: intradermal skin tests, cultures, and guinea-pig inoculations for tuberculosis; bone marrow biopsy and culture; muscle biopsy and culture; cultures for brucella, fungi, and Listeria monocytogenes; agglutina- 


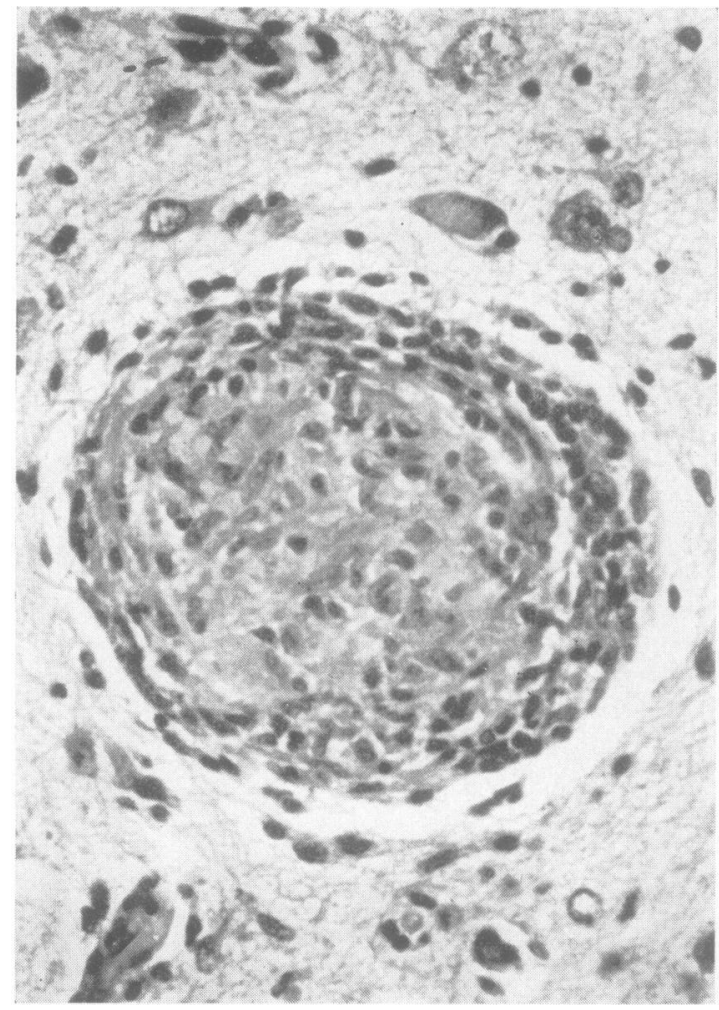

FIG. 1

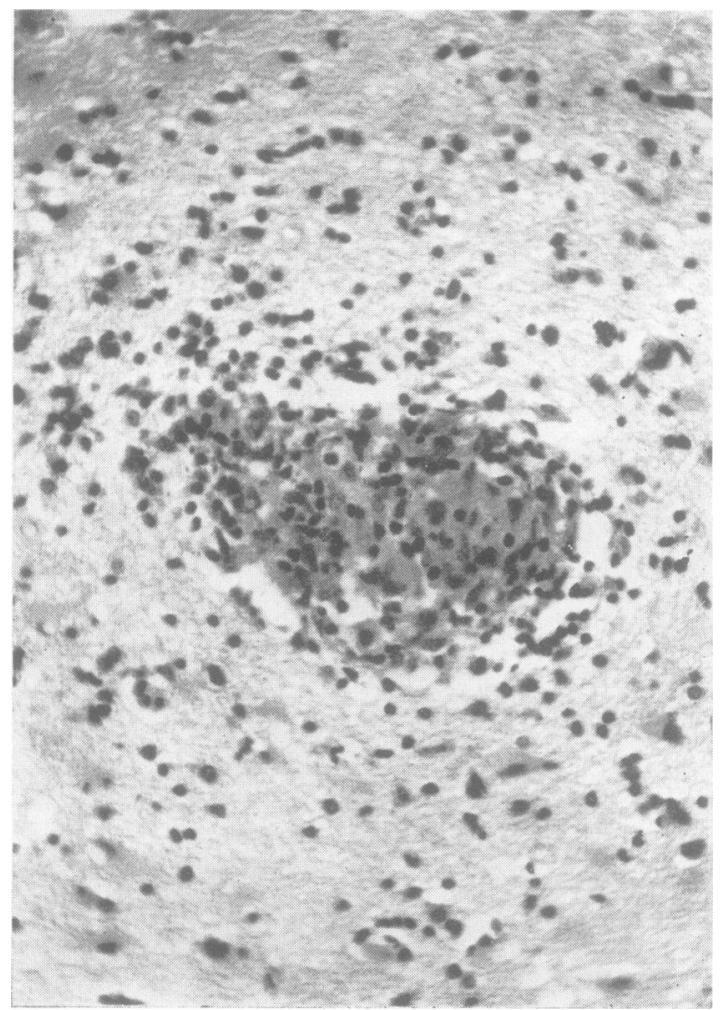

FIG. 2

FIG. 1. Surgical biopsy of the cortex of the right cerebral hemisphere 21 months before death. The circumscribed granulomatous proliferation is producing compression of the adjacent parenchyma. Haematoxylin and eosin, $\times 450$.

FIG. 2. Surgical biopsy of the white matter of the right cerebral hemisphere, 21 months before death. In addition to the granulomatous proliferation, many lymphocytes are evident, spilling over into the adjacent white matter. Haematoxylin and eosin, $\times 370$.

tion tests for histoplasmosis, toxoplasmosis, and listeriosis; Widal and Rickettsia agglutinations, and the Wassermann reaction. The muscle biopsy exhibited a neurogenic type of myopathy. A right scalene lymph node biopsy and skeletal radiographs were negative for sarcoidosis. The only positive finding was the presence of an enlarged lymph node in the upper mediastinum on a chest radiograph.

Seventeen months before death an aching pain began in her left leg. This increased in intensity and was associated with progressive weakness of the left leg followed by weakness of the right leg. On readmission to hospital, examination revealed a sensory deficit of the parietal type on the left side of the body, more marked in the leg than the arm, a left hemiplegia, and weakness of the left side of the face with deviation of the tongue to the left. The patient also had intermittent bouts of mental confusion, delusion, emotional instability, urinary incontinence, and repeated chills with a temperature reaching $104^{\circ} \mathrm{F}$. A progressive loss of knee, ankle, and plantar reflexes bilaterally occurred while she was under observation. The cerebrospinal fluid protein was now $340 \mathrm{mg}$. \% and the white blood cells 150 per c. mm., still predominantly lymphocytes. In view of the biopsy diagnosis, and because of the steadily progressive downhill course with fever, she was treated with streptomycin and isoniazid, sulphadiazine, and achromycin. No change in the progressive deterioration occurred until, five months after this admission to hospital, corticosteroids were administered with remarkable results. Fever promptly subsided and also the pain in her legs, while considerable improvement occurred in her strength, mental state, and general well-being. The power in both upper limbs and the right lower limb increased but an indwelling catheter was still needed. The clinical diagnosis at this time was a left hemiplegia secondary to an intracerebral granulomatous process, and a cauda equina lesion, probably of the same nature.

For the six months preceding her terminal admission to hospital, there was recurrence of the fever together with slight worsening of the neurological picture despite chloromycetin and increased prednisone therapy. When 
admitted to hospital two months before death, there was marked pallor and increased weakness and wasting of the left upper extremity, complete paralysis and wasting of the left leg, reduced power on the right side of the body, and a very labile emotional state. Examination disclosed a mixed urinary infection, and a macrocytic anaemia with a haemoglobin of $5 \mathrm{~g}$. \%. The latter was considered to be secondary to the prolonged anticonvulsant therapy, and the patient responded well to folic acid. Chest radiographs showed enlarged lymph nodes in the mediastinum, and a biopsy from an enlarged lymph node in the left axilla was reported as malignant lymphoma. Finally, because of the poor response to therapy, irradiation to the brain was begun. The course was cut short by a rapid deterioration in her clinical state after the administration of $1,050 \mathrm{r}$ over a three-day period. Terminally she developed a right-sided lower lobar pneumonia, lapsed into coma and died.

\section{PATHOLOGICAL FINDINGS}

The necropsy (NP: 207-59), performed four hours after death, showed severe wasting of the muscles of the left arm and leg. Dissection revealed five moderately enlarged tracheobronchial lymph nodes, the structure of which was partially or completely replaced by a homogeneous grey rubbery tissue. The spleen weighed $145 \mathrm{~g}$. and appeared normal. Bilateral chronic pyelonephritis of moderate severity was present. Microscopically, both the tracheobronchial nodes and the spleen showed the same picture. The normal lymphoid architecture was replaced by a diffuse infiltration of lymphocytes among which were scattered large reticulum cells, a moderate number of Reed-Sternberg cells, plasma cells, and aggregations of eosinophils. Foci of oedema with necrosis and patchy islands of fibrosis were also seen.

No tumour tissue was observed in the spinal canal or cranial cavity in relation to the dura mater. The brain weighed $1,275 \mathrm{~g}$. after fixation in $10 \%$ formalin. The leptomeninges over the lateral surfaces of the frontal lobes, especially on the right side, showed a diffuse grey opacity. The frontal cortex, beneath the thickened meninges on the right side, was slightly depressed over an area measuring 6 by $9 \mathrm{~cm}$. Horizontal and coronal sections through the cerebral hemispheres revealed an irregular confluent replacement of the white matter of the right centrum semiovale by spongy grey tissue which in many areas was depressed below the surrounding cut surface (Fig. 3). This spongy grey tissue extended through the body of the corpus callosum, which was moderately thinned, to involve the white matter in the region of the left cingulate gyrus. In many convolutions there was apparent sparing of the arcuate fibres. In the right cerebral hemisphere the lesion extended into the anterior limb of the internal capsule and into the external capsule with destruction of the claustrum (Fig. 4). Small areas of cystic softening were present in the right putamen. The anterior horn of the right lateral ventricle showed slight dilatation. Posteriorly, the spongy degeneration in the centrum semiovale did not extend beyond the splenium of the corpus callosum. The basal cerebral arteries and proximal portions of the anterior and middle cerebral arteries appeared normal. However, small leptomeningeal branches of the right middle cerebral artery overlying the insular cortex showed diffuse thickening of their walls, with resulting moderate to severe stenosis of their lumina (Fig. 4). No changes were seen in the cerebellum, brain-stem, spinal cord, or spinal nerve roots, on gross examination.

For microscopic study, blocks were embedded in paraffin and celloidin or cut on a freezing microtome. The following staining procedures were used: on paraffinembedded material, haematoxylin and eosin, Mallory's connective tissue stain, cresyl violet, phosphotungstic acid haematoxylin, Bodian stain for axons, Luxol fast blue and Smith and Quigley for myelin sheaths, Weigert stain for elastic tissue, periodic-acid-Schiff and Gram stains. Loyez and Gros Bielschowsky stains were performed on celloidin-embedded tissue, and Scharlach $\mathbf{R}$, Cone and Penfield's silver carbonate for oligodendroglia and microglia, and victoria blue for astrocytes, on frozen sections.

Histologically, there was a varied structure to the area of spongy degeneration in the white matter of the right cerebral hemisphere. In many regions the predominant finding was a loss of myelin sheaths with a relative sparing of the axons (Figs. 5 and 6). In other areas the parenchyma was completely destroyed with focal areas of cystic degeneration. Sparing of arcuate fibres was often seen. Throughout these lesions were abundant hypertrophied astrocytes with long thick glial processes and large vesicular nuclei. Occasional binucleate forms were present but no astrocytes in mitosis or large cells with collections of micronuclei were found. There were no intranuclear or intracytoplasmic inclusion bodies. The oligodendroglia were moderately reduced in number, but those that remained were normal in appearance. Numerous microglial cells with sudanophilic material in their cytoplasm were seen throughout. Both descending corticospinal tracts showed demyelination and gliosis, these changes being very severe in the tract originating in the right cerebral hemisphere.

The proximal portions of the right middle cerebral artery and the right and left anterior cerebral arteries showed a few focal areas of minimal intimal fibrous thickening, with normal underlying media and adventitia. However, numerous small leptomeningeal and perforating branches of these vessels exhibited severe stenosis or occlusion of their lumina. These changes were focal in nature, and were of varying ages. Serial sections showed the active lesions to begin in the adventitial layer as a proliferation of histiocyte-like cells. Some of the cells coalesced to form multinucleated giant cells. These proliferating cells encroached upon the media to varying degrees, while a concentric fibrocellular thickening occurred in the intima. In the most severe lesions, there was complete involvement of the arterial wall (Fig. 7), complete or partial fragmentation and disappearance of the internal elastic lamina (Fig. 8), and stenosis of the lumen with or without occlusion by thrombus. The older vascular lesions showed stenosis or occlusion of the lumen, varying degrees of hyalinization of the intima and media, intense crenation and areas of reduplication 


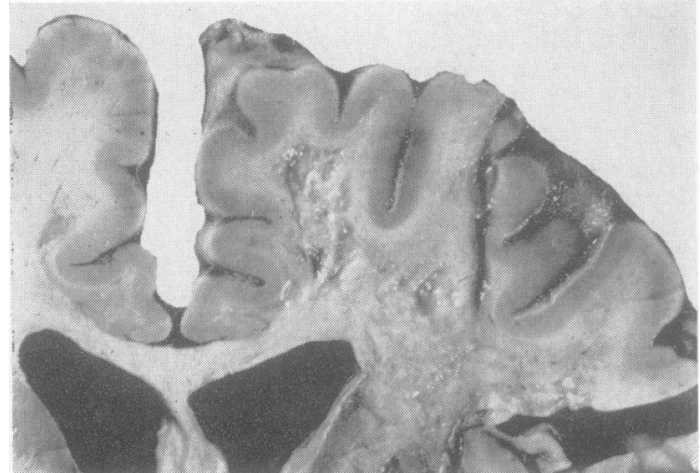

FIG. 3

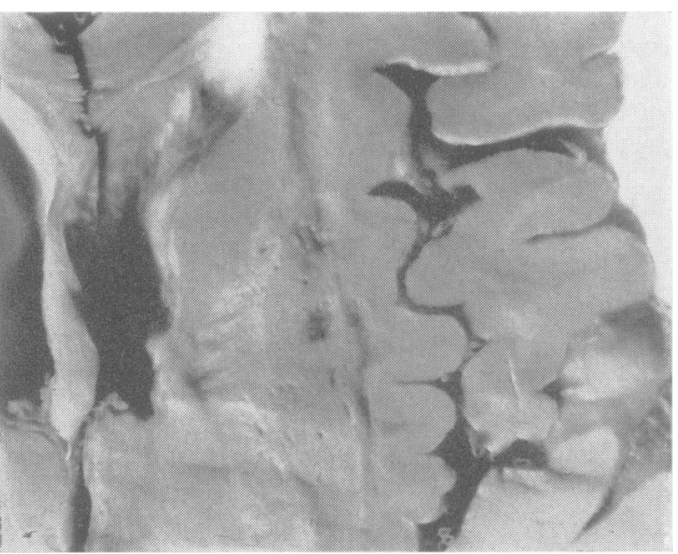

FIG. 4

FIG. 3. Coronal section through the right cerebral hemisphere shows the typical appearance of the spongy degeneration in the right centrum semiovale. The linear scar passing vertically through the cortex and white matter represents the site of the surgical biopsy 21 months before death.

FIG. 4. Horizontal section through the region of the right basal ganglia shows degeneration in the external capsule and focal areas of cystic degeneration in the putamen.

FIG. 5. Coronal section through the right cerebral hemisphere shows an irregular loss of myelin with relative sparing of arcuate fibres. Loyez, $\times 1 \frac{1}{4}$.

FIG. 6. Residual axons in the areas of demyelination shown in Fig. 5. The large cells are hypertrophied astrocytes, while apparently normal oligodendroglia and phagocytic microglia are also present. Bodian, $\times 300$.

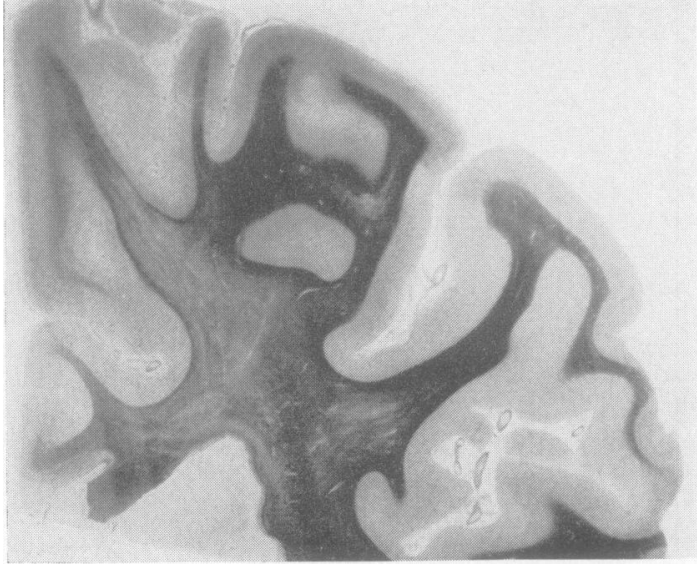

FIG. 5

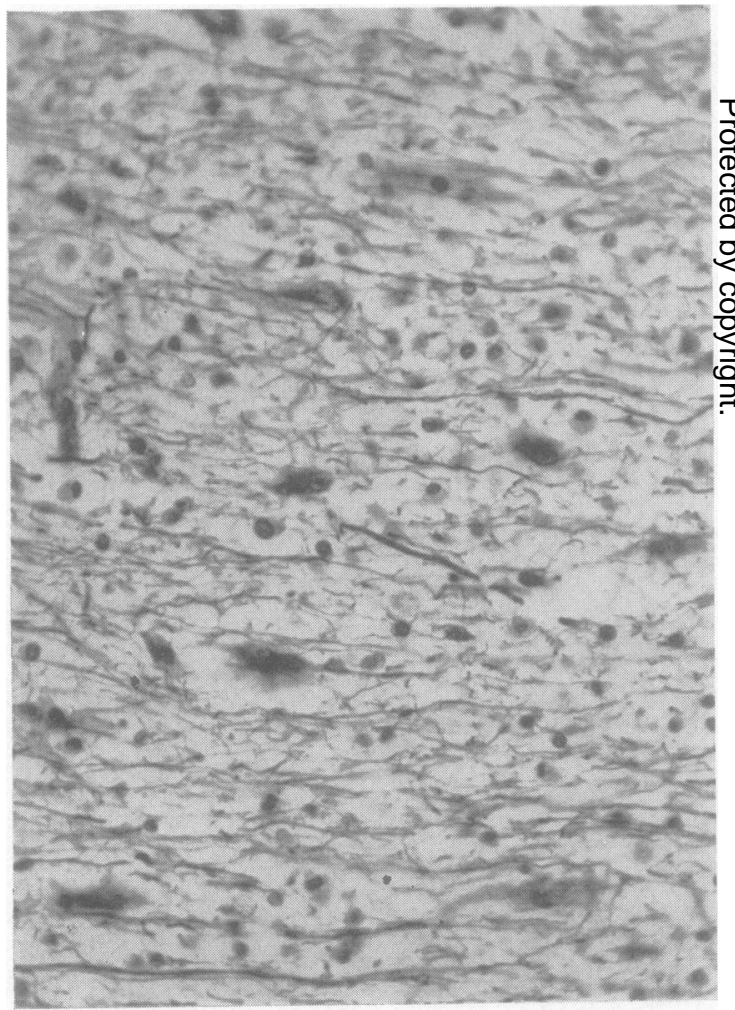

FIG. 6 


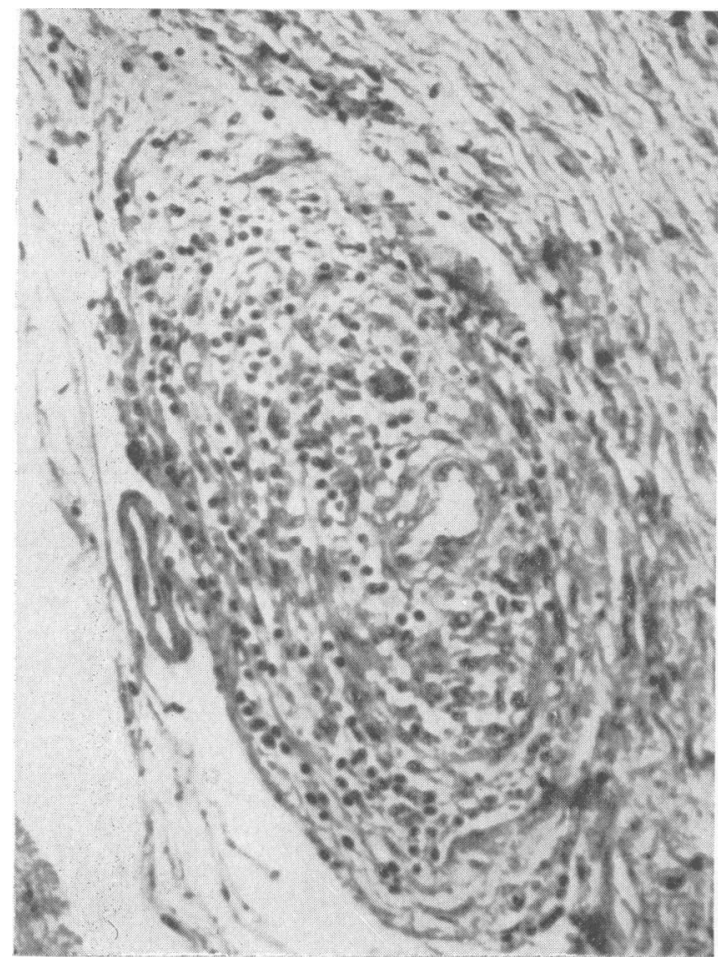

FIG. 7

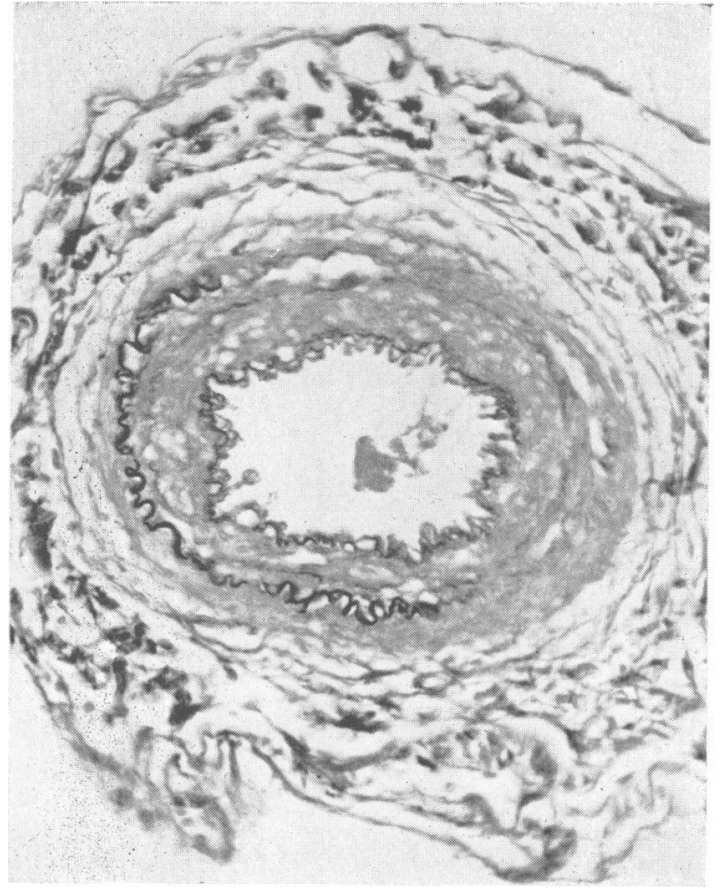

FIG. 9

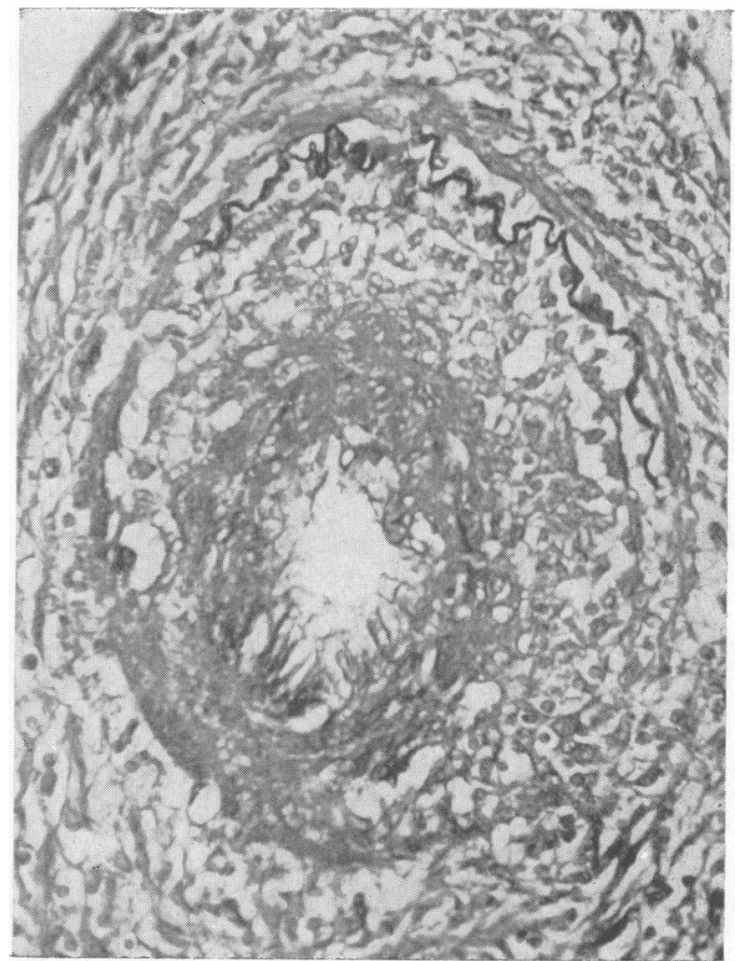

FIG. 8

FIG. 7. This section shows an active cellular proliferation involving all layers of the wall of a small leptomeningeal artery. Haematoxylin and eosin, $\times 200$.

FIG. 8. Evidence of active cellular vascular proliferation. Only a few residual muscle fibres remain in the media, and large segments of the internal elastic lamina are destroyed. Weigert's elastic, $\times 250$.

FIG. 9. An old lesion of the wall of a leptomeningeal artery with hyalinization of the media, permanent damage to the internal elastic lamina, and reduplication of elastic fibres in the intima. Weigert's elastic, $\times 300$. 


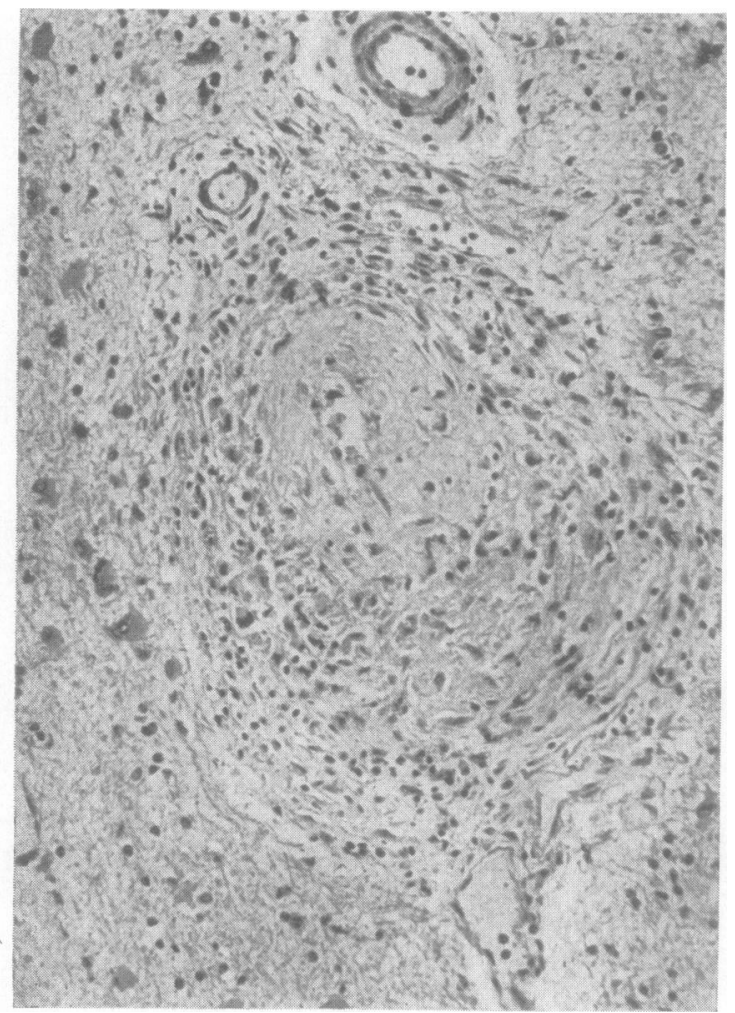

FIG. 10

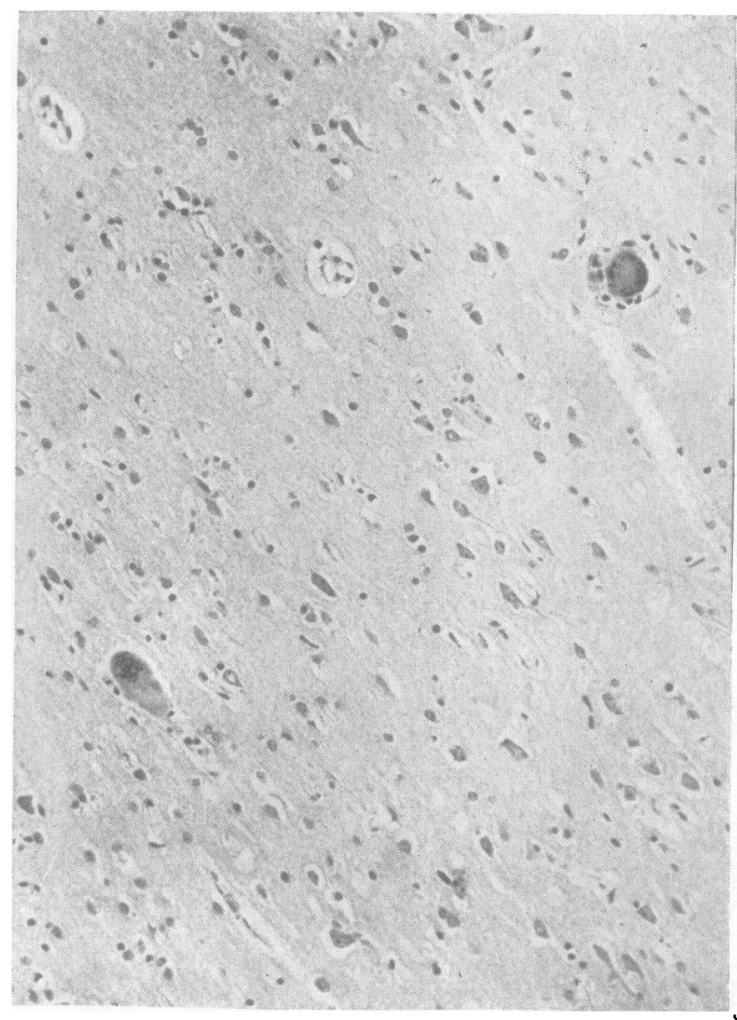

FIG. 11

FIG. 10. Severe involvement of the wall of a small artery of the right anterior perforated substance. Extensive deposition of acellular hyalin material is seen. Haematoxylin and eosin, $\times 140$.

FIG. 11. Multinucleated giant cells in the cortex overlying an area of spongy degeneration. These cells are in intimate? relation to small vessels and are not seen free within the brain parenchyma. Haematoxylin and eosin, $\times 135$.

of the internal elastic lamina, often with segmental defects (Fig. 9). These vascular lesions were most numerous in the subarachnoid space adjacent to convolutions showing extensive degeneration in the underlying white matter. However, in addition to the vascular involvement in the subarachnoid space, numerous lesions, both active and old, were present in the arteries traversing the right anterior perforated substance. Fig. 10 shows one of these vessels with extensive hyalinization of its wall. Less intense, but similar changes were seen involving the anterior and posterior spinal arteries and small vessels within the brain-stem.

Granulomas of similar morphology to those seen in the surgical biopsy were not found in the necropsy material. However, in the relatively normal cortex overlying the areas of spongy degeneration were a number of multinucleated giant cells (Fig. 11). These cells were found in smaller numbers in the damaged white matter. They were intimately related to the walls of arterioles, capillaries, and venules. Perivascular giant cells were present occasionally in the brain-stem but were numerous in the dorsal and ventral nerve roots of the lower lumbar and sacral segments of the spinal cord. In this situation they were often associated with perivascular foci of histiocytes, lymphocytes, and the occasional plasma cell. Macrophages containing intracytoplasmic droplets of sudanophilic material were found in the dorsal sacral nerve roots, and demyelination of these roots had resulted in a severe ascending bilateral demyelination of the fasciculus gracilis. There was demyelination of the left sciatic nerve considered to be secondary to lesions in the ventral nerve roots.

\section{DISCUSSION}

The microscopical appearance of the tissue from the centrum semiovale of the cerebral hemispheres showed a wide variation. In some areas it was very suggestive of a primary demyelinating disease with loss of myelin sheaths and reduction of oligodendroglia, while axons were relatively spared. However, 
in many other areas, the tissue showed changes consistent with old ischaemic softening. Though predominantly involving white matter, focal areas of old softening were seen in the putamen. The preservation of the overlying cerebral cortex was an outstanding feature. The focal areas of stenosis or occlusion in the small leptomeningeal vessels were abundant in the vicinity of the most extensive parenchymal lesions. The granulomatous proliferations involving the walls of the small vessels of the parenchyma, observed in the biopsy 21 months before death, were not seen. Perivascular multinucleated giant cells were present, both in the cortex, and to a lesser extent in the white matter surrounding the damaged areas. The focal lesions in the walls of small leptomeningeal vessels, which appeared to be actively proliferating at the time of death, did resemble those of the biopsy material.

The predilection of this focal granulomatous proliferation for the vessels of the right cerebral hemisphere, with rarefaction and necrosis of tissue, undoubtedly resulted in the left-sided clinical symptoms and signs. Encroachment upon the white matter of the medial part of the left centrum semiovale probably accounted for the later clinical involvement of the right leg. Similar vascular lesions in the dorsal and ventral roots of the lower lumbar and sacral segments of the spinal cord, with ascending demyelination in the fasciculus gracilis, and demyelination of peripheral nerves, resulted in the development of the cauda equina syndrome.

Åströ.n, Mancall, and Richardson (1958) drew attention to changes occurring within the central nervous system in three cases of malignant lymphoma. They described these changes as a separate disease entity which they called progressive multifocal leucoencephalopathy. They found five cases in the literature which would fit into this group. Since then, four other cases have been described (Cavanagh, Greenbaum, Marshall, and Rubinstein, 1959; Lloyd and Urich, 1959; Dolman and Cairns, 1961). Of all these, five were associated with Hodgkin's disease. Histologically, though our case showed numerous hypertrophied astrocytes, we did not find the large monster astrocytes, the enlarged oligodendroglia with homogenization of nuclear chromatin nor the acidophilic intranuclear inclusions which characterize the above cases of progressive multifocal leuco-encephalopathy.

Cravioto and Feigin (1959) described two cases of their own and discussed six others from the literature, in which there were multifocal lesions in the walls of small blood vessels throughout the central nervous system. These cases were not associated with malignant lymphoma. They were characterized by a granulomatous proliferation involving the walls of small arteries and veins. All eight cases showed the central nervous system to be the site of predilection for the vascular lesions. In their case 2 , and in one of the cases from the literature, there was also involvement of vessels in other organs of the body, but to a much lesser degree. These authors suggested that this group represents a distinct clinical and pathological entity and that the disease process should be designated as 'non-infectious, granulomatous angiitis with a predilection for the nervous system'. In these reported eases only rarely was the full thickness of the vessel wall involved. In the majority of instances the granulomatous proliferation was confined to the adventitial and intimal layers with sparing of the media. We did not see sparing of the media of the small vessels in our case, and, as described, the granulomatous proliferation appeared to begin in the adventitia and extend into the media, while the intima showed a fibrocellular thickening, with or without occlusion of the lumen by thrombus

The association of encephalitis with Hodgkin's disease is uncommon, although occasional cases have been described (Askanazy, 1921). Such cases invariably exhibited lymphocytic cuffing of the sm: vessels of the meninges and the brain parenchyma Rottino and Hoffman (1950) described a case of Hodgkin's disease associated with a predominantly granulomatous angiitis of the small vessels of the brain and meninges. They interpreted these changes as a sarcoid form of encephalitis. From the descriptions and photographs presented by Cravioto and Feigin and by Rottino and Hoffman we consider their cases similar to the one described in this paper.

The fundamental changes in our case are those of a granulomatous angiitis showing a predilection for the small vessels of the right cerebral hemisphere, and for the small arteries and veins at the lower end of the spinal cord. However, the occasional involved vessel was seen throughout all areas of the central nervous system. The degenerative changes in the parenchyma of the right cerebral hemisphere and in the nerve roots of the lower end of the spinal cord were considered to be secondary to the vascular damage. The relative sparing of the cerebral cortex may be explained by the development of a collateral circulation. Unfortunately, the active cellular proliferation seen in the biopsy material was not encountered 21 months later at the time of death. The depressant action of the administered corticosteroid hormones on the reticulo-endothelial system would possibly explain the absence of these proliferative lesions. Residual sites of cellular proliferation would perhaps be indicated by perivascular giant cells in the cortex and white matter, and by perivascular collections of histiocytes in the spinal 
cord and the dorsal and ventral nerve roots of the lower lumbar and sacral segments.

The question of a hypersensitivity reaction as the basis of these vascular lesions is a moct one. Clinical cases of Hodgkin's disease occasionally show an associated anomaly of their immunological mechanism, such cases often presenting with circulating plasma haemolysins. The granulomatous reaction seen in the biopsy material in this case very closely resembles the granulomatous angiitis of experimental allergic encephalomyelitis described by many investigators. The distribution is also similar, being confined mainly to the central nervous system and, to a lesser extent, the dorsal and ventral nerve roots. Cameron, Howell, and Hutchinson (1958) described a case of an acute peripheral neuropathy associated with Hodgkin's disease. There was no disease of the central nervous system. The vascular lesions in their case were acute, consisting of perivascular cuffing with lymphocytes and plasma cells, which they likened to the reaction seen in experimental allergic neuritis produced by Waksman and Adams $(1955,1956)$ in rabbits, guinea-pigs, and mice.

\section{SUMMARY}

The case of a 31-year-old white woman has been described. She presented clinically with a progressive neurological disorder, during the course of which a cerebral biopsy revealed focal granulomatous angiitis. Late in her illness Hodgkin's disease was diagnosed by lymph node biopsy. The basic pathological change was a multifocal granulomatous angiitis involving small arteries and veins of the central nervous system. The ensuing stenosis and occlusion of blood vessels resulted in damage to the brain and the roots of the lower lumbar and sacral segments of the spinal cord, the latter giving rise clinically to a cauda equina syndrome.

We wish to tender our appreciation to Drs. K. J. R. Wightman and H. J. Barrie for making available the $\vec{F}$ clinical and general necropsy records and to Dr. J. Olszewski for his helpful criticism of this manuscript.

\section{REFERENCES}

Askanazy, M. (1921). Verh. dtsch. path. Ges., 18, 78.

Áström, K-E., Mancall, E. L., and Richardson, E. P., Jr. (1958). Brain, 81, 93.

Cameron, D. G., Howell, D. A., and Hutchinson, J. L. (1958) Neurclogy, 8, 575.

Cavanagh, J. B., Greenbaum, D., Marshall, A. H. E., and Rubinstein L. J. (1959). Lancet, 2,524

Cravioto, H., and Feigin, I. (1959). Neurology, 9, 599.

Dolman, C. L., and Cairns, A. R. M. (1961). Ibid., 11, 349.

Ginsburg, S. (1927). Arch. intern. Med., 39, 571.

Hutchinson, E. C., Leonard, B. J., Maudsley, C., and Yates, P. O. N (1958). Brain, 81, 75.

Lloyd, O. C., and Urich, H. (1959). Lancet, $2,529$.

Rottino, A., and Hoff.nan, G. (1950). J. Neuropath. exp. Neurol., 90 103.

Sparling. H. J., Jr., Adams, R. D., and Parker, F., Jr. (1947). Medicin (Baltimore), 26, 285.

Waksman, B. H., and Adams, R. D. (1955). J. exp. Med., 102, 213.

- (1956). J. Neuropath. exp. Neurol., 15, 293.

Winkelman, N. W., and Moore, M. T. (1941). Arch. Neurol. Psychiat (Chicago), 45, 304. 